\title{
A systematic review and meta-analysis of nab-paclitaxel mono-chemotherapy for metastatic breast cancer
}

\author{
Haili Lu \\ Changzheng Hospital: Shanghai Changzheng Hospital \\ Siluo Zha \\ Changzheng Hospital: Shanghai Changzheng Hospital \\ Wei Zhang \\ Changzheng Hospital: Shanghai Changzheng Hospital \\ Qiang Wang \\ Changzheng Hospital: Shanghai Changzheng Hospital \\ Daozhen Jiang \\ Changzheng Hospital: Shanghai Changzheng Hospital \\ Xinyun Xu \\ Changzheng Hospital: Shanghai Changzheng Hospital \\ Xiangmin Zheng \\ Changzheng Hospital: Shanghai Changzheng Hospital \\ Ming Qiu \\ Changzheng Hospital: Shanghai Changzheng Hospital \\ Chengxiang Shan ( $\sim$ chengxiangshan@smmu.edu.cn ) \\ Department of General Surgery of Changzheng Hospital affiliated to Naval Military Medical University
}

\section{Research article}

Keywords: nab-paclitaxel, metastatic breast cancer, safety, efficacy, meta-analysis

Posted Date: December 23rd, 2020

DOl: https://doi.org/10.21203/rs.3.rs-132448/v1

License: @ (1) This work is licensed under a Creative Commons Attribution 4.0 International License. Read Full License

Version of Record: A version of this preprint was published at BMC Cancer on July 18th, 2021. See the published version at https://doi.org/10.1186/s12885021-08441-z. 


\section{Abstract \\ Background}

Various clinical trials and real-life studies have tried to explore the value of nab-paclitaxel mono-chemotherapy for metastatic breast cancer (MBC). The safety and efficacy of nab-paclitaxel needs to be systematically evaluated.

\section{Methods}

Electronic searches for prospective clinical trials containing nab-paclitaxel monotherapy for MBC were performed. Requisite data were extracted, integrated and analyzed from the included studies according to different purposes using systematic review and meta-analysis.

\section{Results}

22 studies with 3287 MBC patients were included. 1685 MBC patients received nab-paclitaxel as first-line therapy, 640 patients as further-line therapy, and 962 patients as mixed-line therapy. $1966 \mathrm{MBC}$ patients (60.40\%) received nab-paclitaxel weekly, while 1190 patients (36.56\%) received nab-paclitaxel triweekly and 99 patients (3.04\%) biweekly. The overall incidence of all grades neutropenia, leukopenia, peripheral sensory neuropathy, and fatigue was $52 \%$ ( $95 \% \mathrm{Cl}$, $38 \%-66 \%$ ), 58\% (95\% Cl, 43\%-73\%), 58\% (95\% Cl, 48\%-68\%), and 49\% (95\% Cl, 41\%-56\%) respectively. The overall response rate (ORR) was 40\% (95\% $\mathrm{Cl}$, $35 \%-45 \%)$ and the clinical benefit rate (CBR) was $66 \%$ (95\% Cl, 59\%-73\%) following nab-paclitaxel monotherapy. The median progression free survival (PFS) was 7.64 months ( $95 \% \mathrm{Cl}, 6.89-8.40$ months) and the median overall survival (OS) was 24.51 months (95\% Cl, 21.25-27.78 months). According to the metaregression analysis, grade $3 / 4$ neutropenia occurred less frequently in Her-2 negative patients compared with all population ( $P=0.046)$. Patients who received first-line nab-paclitaxel monotherapy showed higher ORR $(P=0.006)$ and longer PFS $(P=0.045)$. Patients who received further-line therapy was demonstrated to have shorter median OS versus first- and mixed-line therapy. Efficacy outcomes were not affected by the administration schedule. However, patients appeared to have more superior ORR $(P=0.044)$ and longer PFS $(P=0.03)$ along with the increasing dosage of nab-paclitaxel under the same schedule.

\section{Conclusions}

Both weekly and triweekly nab-paclitaxel mono-chemotherapy were proved to be effective for MBC with generally reasonable toxicity profiles. Higher ORR, longer PFS and OS would be achieved in patients treated with nab-paclitaxel as first line. Increasing nab-paclitaxel dosage would result in better tumor control (higher ORR and PFS). Changing nab-paclitaxel schedule had no benefit on ameliorating the overall survival.

\section{Background}

Approximately one fourth of patients with earlier localized breast cancer will eventually develop recurrent or metastatic breast cancer (MBC) [1]. Once breast cancer becomes metastatic it is rarely curable, even if mortality has been decreasing steadily in the developed countries in the last decade [2]. Although no randomized evidence exists comparing therapy with observation in women with $\mathrm{MBC}$, it is widely recommended that women with $\mathrm{MBC}$ should receive some form of systemic therapy during the course of their disease [3]. Chemotherapy has been the cornerstone in the treatment of MBC patients for many years, and it is generally accepted that taxanes are among the most active single agents [4].

The clinical approval of taxanes for MBC began with paclitaxel in 1994, continued with docetaxel in 1996, and still updated with nanoparticle albumin-bound paclitaxel (Abraxane, nab-paclitaxel) in 2005 [5]. Although paclitaxel and docetaxel had proven to be clinical beneficial, their hydrophobic chemical formulations had presented obvious limitations [6]. Nab-paclitaxel was developed to eliminate the solvent-related toxicities typically associated with taxanes administration. More importantly, this colloidal suspension was also designed to preferentially deliver paclitaxel to tumors by biologically interacting with albumin receptors that mediated drug transport [7]; in vitro studies had demonstrated a 4.5 -fold increase in paclitaxel transport across endothelial cells for nab-paclitaxel compared to conventional taxanes [8].

Since Ibrahim NK firstly reported $300 \mathrm{mg} / \mathrm{m} 2$ nab-paclitaxel monotherapy in a triweekly style resulting in a $48 \%$ overall response rate (ORR) for $63 \mathrm{MBC}$ patients in a phase II trial [9], various clinical trials and real-life studies tried to explore the safety and activity of nab-paclitaxel in treating MBC. Most recently, in the NABUCCO observational study Marschner N reported the nab-paclitaxel monotherapy could offer a 37.2\% ORR, a $68.3 \%$ clinical benefit rate (CBR), a 5.9 months of median time to progression (TTP) and a 15.6 months of median overall survival (OS) with lower (5\%) grade $3 / 4$ treatment-related adverse events (TRAEs) in $697 \mathrm{MBC}$ patients[10]. Head-to-head clinical comparisons between nab-paclitaxel and conventional taxanes were not lacking, with two pivotal phase II/III clinical trials reported by Gradishar WJ [11, 12], coincidentally demonstrated superior efficacy and safety of nab-paclitaxel compared with paclitaxel $(175 \mathrm{mg} / \mathrm{m} 2, \mathrm{q} 3 \mathrm{w})$ or docetaxel $(100 \mathrm{mg} / \mathrm{m} 2, \mathrm{q} 3 \mathrm{w})$, with a statistically higher ORR and clinically significant prolongation of progression-free survival (PFS) with shorter infusion schedules (30 minutes) and no premedication. However, opposite results still existed. In a phase II multicenter trial with 197 Her2 negative MBC, Tamura K reported similar efficacy outcomes in patients treated with weekly nab-paclitaxel (150 mg/m2) and triweekly docetaxel (75 mg/m2). Nab-paclitaxel did not show superiority in PFS compared with docetaxel [13]. In the CALGB 40502 study, Rugo HS also failed to demonstrate superiority of nab-paclitaxel given weekly compared with paclitaxel in $542 \mathrm{MBC}$ patients with increased overall toxicity. They suggested weekly paclitaxel should remain the preferred microtubule inhibitor for treating patients with MBC in the first-line setting [14]. Therefore, considering a proportion of studies on nab-paclitaxel monotherapy were single-arm, non-randomized phase II trials with rather small sample size, the safety and efficacy of nab-paclitaxel needed to be excavated deeply and thoroughly. 
Although nab-paclitaxel was initially approved by US Food and Drug Administration (FDA) with a recommended triweekly $260 \mathrm{mg} / \mathrm{m} 2 \mathrm{dosage}$ for MBC, evidence suggested that a weekly nab-paclitaxel regimen could also be feasible for patients with MBC inspired by weekly paclitaxel administration appearing to be the optimal schedule for MBC [7]. In fact, a retrospective study reported by Dent S showed inferior ORR (4.7\% vs. $14.3 \%)$, CBR ( $57.1 \%$ vs. $76.2 \%)$, and shorter median OS (10.8 months vs. 13.6 months) in the triweekly nab-paclitaxel group compared with weekly nab-paclitaxel group [15]. Gradishar WJ also demonstrated better disease control for nab-paclitaxel qw 3/4 regimens (100 mg/m2 and $150 \mathrm{mg} / \mathrm{m} 2)$ versus a q3w regimen as monotherapy, and nabpaclitaxel at $150 \mathrm{mg} / \mathrm{m} 2$ qw $3 / 4$ resulted in a 33.8 months OS longer than historically achieved with single-agent taxanes therapy in MBC [16]. However, in the NABUCCO study, efficacy superiority with respect to better tumor control and longer survival outcomes had not been obtained in the weekly nab-paclitaxel group [10]. Irrespective of the survival outcomes, weekly nab-paclitaxel regimen seemed to increase paclitaxel-related toxicity. Tamura $\mathrm{K}$ reported $150 \mathrm{mg} / \mathrm{m} 2$ qw 3/4 nab-paclitaxel monotherapy would result in 78\% of grade 3/4 neutropenia and 22\% of grade 3/4 neuropathy in 98 MBC patients [13]. Decreased quality of life (QOL) due to more TRAEs in MBC patients seemed to disobey the goal of MBC treatment [17]. Since no tailored regimens were strongly recommended, variable studies with different nab-paclitaxel dosage and schedule came into clinical practice [18], and these data called into question which regimen of nab-paclitaxel was the optimal setting for MBC.

To date, nab-paclitaxel had seemed been suggested quite important as a single agent for first-line or further-line treatment of MBC [19]. Since it was hard to interpret all the information into a clear conclusion in terms of the value of nab-paclitaxel in metastatic setting due to the limitation of every single study, here we reviewed the clinical evidence with nab-paclitaxel as a single agent in metastatic settings treatment, with the goal of understanding the safety and efficacy followed by nab-paclitaxel mono-chemotherapy. Furthermore, evidence-based optimal regimen and schedule of nab-paclitaxel for MBC was also explored by meta-regression analysis and subgroup analysis.

\section{Methods}

Study search strategy

According to the Preferred Reporting Items for Systematic Reviews and Meta-Analyses (PRISMA) guideline [20], a systematic search was independently performed by 2 investigators (Shan CX and Lu HL) using electronic databases including PubMed/Medline, ClinicalTrial.gov and the Cochrane Center Register of Controlled Trials to identify articles published between March 2005 and March 2020, using the following search keywords :"nab-paclitaxel or albuminbound paclitaxel or abi-007 or Abraxane, and metastatic breast cancer". The "related articles" function was used to broaden the search. All abstracts, studies and citations were checked for additional material when appropriate. In addition, abstracts from annual meetings of the American Society of Clinical Oncology (ASCO), European Society of Medical Oncology Conference (ESMO) and San Antonio Breast Cancer Symposium (SABCS) were retrieved for relevant abstracts using similar search terms. No language restrictions were made.

Study selection criteria

Abstracts or full-text articles were initially screened, and then selected or rejected by the two reviewers (Shan CX and Lu HL) on the basis of the inclusion and exclusion criteria described below.

Inclusion criteria: (1) Designed prospective trials, including both observational studies and interventional studies. (2) Phase II clinical trials, phase III clinical trials and cohort studies. (3) Single arm, two arms or multi-arms trials which contained the treatment group of nab-paclitaxel monotherapy were all included. (4) The exact data of dichotomous-type information and continuous-type information, by which the standard deviation or standard error could be calculated, should be provided so as to integrate each single weight in each study.

Exclusion criteria: (1) Retrospective observational studies. (2) Total sample capacity < 10 cases. (3) Studies with the same research subjects published repeatedly by different journals.

Quality assessment

Methodological quality of randomized controlled trials (RCTs) was assessed by Cochrane Collaboration tool with six domains: random sequence generation, allocation concealment, blinding of investigators, participants and outcome assessor, completion of outcome data, selective reporting. All of the domains should be graded as "low risk", "high risk" or "unclear risk" of bias. If no less than 4 "low risk" domains were identified in the trial with none "high risk" domain, the trial was considered with low risk and high quality.

The Newcastle-Ottawa Scale was used to evaluate the quality of cohort study. Grading criteria included: representativeness of the exposed cohort, selection of the nonexposed cohort, ascertainment of exposure, demonstration that outcome of interest was not present at start of study, comparability of cohorts on the basis of the design or analysis, assessment of outcome, enough time to follow up, adequate follow-up. The maximum score was nine.

For non-randomized trials, quality assessment was performed by Methodological Index for Non-randomized Studies (MINORS). For trials without control group, eight criteria were required for evaluation: clear object, consecutive participants, collection of expected result, terminal point could properly reflect the purpose, objectivity of endpoint evaluation, long enough to follow up, the rate of lost to follow up was less than $5 \%$, estimation of sample size. For those with control group, four additional criteria were also required: appropriate choice of control group, patients were selected at the same period, comparability of two groups, optimal statistical analysis. The criteria were graded from zero to two points according to the information reported rarely, inadequately or in detail. The maximum score was sixteen or twenty-four.

Data extraction 
The two reviewers independently extracted details from each eligible study which comprised (1) information and quality of the research: first author, year of publication, study design, treatment line, population, sample capacity; (2) nab-paclitaxel regimen including the dosage and schedule; (3) The assessment data of trials containing multiple groups were initially divided into single nab-paclitaxel mono-chemotherapy group and other groups, and then extracted individually; (4) toxicity profile, including the incidence of all grades and grade 3/4 of neutropenia, leukopenia, peripheral sensory neuropathy and fatigue; (5) disease control rate, including ORR and CBR; (6) survival endpoints, including median PFS and median OS. Specifically, the assessment data of repeated trials published in different journals at different time was extracted based on the latest and the most detailed article. Furthermore, if the safety and efficacy outcomes were both assessed by radiologist and investigator independently in certain studies, we retrieved the data provided by the investigator.

\section{Statistical analysis}

All trials referring to nab-paclitaxel monotherapy was firstly split into each individual group according to nab-paclitaxel administration dosage and schedule. The outcome measures included the incidence of TRAEs, the disease control rate (ORR, CBR), and median survival time (median PFS, median OS). Data analysis in our study was performed with STATA (version 14.0) software. For the ratio analysis, we performed single-arm meta-analysis and adopted the "metaprop" command set in STATA for data integration. Additional "ftt" command set was applied if the level of rate was unusual high or low. For the survival outcome analysis, median PFS or median OS with its sampling error were utilized for data integration. The sampling error could be retrieved through the literature data base, while, it could also be calculated with the formula of the upper bound minus the lower bound of $95 \%$ confidence interval (Cl)/2* 1.96 . If significant heterogeneity did exist between the recruited studies ( $2>50 \%)$, we adopted a random-effects model, if not, a fixed-effects model was applied. The publication bias was checked through "Begger" and "Egger" method in STATA, and further represented through funnel plot analysis. We performed metaregression analysis to demonstrate the potential risk factors affecting each single outcome of nab-paclitaxel monotherapy after removing the included studies with significant heterogeneity. If the risk factor did exist, meta-regression subgroup analysis was further performed to demonstrate the difference between individual groups due to the specified risk factor.

\section{Results}

Identification and characteristics of studies

We ultimately identified 22 independent studies published between March 2005 and March 2020 [9, 10, 12, 13, 16, 21, 22, 23, 24, 25, 26, 27, 28, 29, 30, 31, 32, $33,34,35,36,37$. A flow chart representing selection of studies is shown in Figure 1. Totally 11 RCTs, 10 non-randomized trials and 1 cohort study were included. The quality of 11 RCTs were all demonstrated with low risk (Grade A). The only one cohort study was also evaluated as high quality with full score. Based on the criteria of MINORS, five non-randomized trials scored zero point in sample size estimation criteria for bare report (Ibrahim NK 2005, Hurria A 2015, Blum JL 2007, Marschner N 2018, Bernardo A 2017), one trial scored one point for inadequate information (Yamamoto S 2017), and another one trial (Bernardo A 2017) also scored one point in follow-up domain for higher rate ( $>5 \%$ ) of lost to follow-up. The details of study quality were shown in Table 1 . The baseline characteristics of the included studies for metastatic breast cancer were summarized in Table 2 .

Table 1 Quality assessment of the included studies 


\begin{tabular}{|c|c|c|c|c|c|}
\hline Author & Year & Cochrane & NOS & $\begin{array}{l}\text { MINORS } \\
\text { non-control }\end{array}$ & $\begin{array}{l}\text { MINORS } \\
\text { control }\end{array}$ \\
\hline Gradishar WJ & 2005 & Grade A & & & \\
\hline GUAN ZZ & 2009 & Grade A & & & \\
\hline Gradishar WJ & 2012 & Grade A & & & \\
\hline Ranade AA & 2013 & Grade A & & & \\
\hline Andres FT & 2015 & Grade A & & & \\
\hline Jain MM & 2016 & Grade A & & & \\
\hline Tamura K & 2017 & Grade A & & & \\
\hline Gennari A & 2018 & Grade A & & & \\
\hline Ciruelos E & 2019 & Grade A & & & \\
\hline Hara F & 2019 & Grade A & & & \\
\hline Schmid P & 2019 & Grade A & & & \\
\hline Brezden B & 2013 & & 9 & & \\
\hline Ibrahim NK & 2005 & & & 14 & \\
\hline Mirtsching B & 2011 & & & 16 & \\
\hline Fabi A & 2015 & & & 16 & \\
\hline Hurria A & 2015 & & & 16 & \\
\hline Palumbo R & 2015 & & & 16 & \\
\hline Yamamoto S & 2017 & & & 15 & \\
\hline Hurria A & 2019 & & & 14 & \\
\hline Blum JL & 2007 & & & & 22 \\
\hline Bernardo A & 2017 & & & & 21 \\
\hline Marschner N & 2018 & & & & 22 \\
\hline
\end{tabular}

Table 2 Baseline characteristics of the included studies for metastatic breast cancer 


\begin{tabular}{|c|c|c|c|c|c|c|c|c|c|c|c|c|c|}
\hline \multirow[t]{4}{*}{ Author+Year } & \multirow{4}{*}{$\begin{array}{l}\text { Study } \\
\text { Design }\end{array}$} & \multirow{4}{*}{$\begin{array}{l}\text { Sample } \\
\text { Size } \\
\text { (N) }\end{array}$} & \multirow{4}{*}{$\begin{array}{l}\text { Treatment } \\
\text { Line }\end{array}$} & \multirow{4}{*}{$\begin{array}{l}\text { Nab- } \\
\text { paclitaxel } \\
\text { Monotherapy } \\
\text { Regimen }\end{array}$} & \multicolumn{8}{|c|}{ Treatment Related Adverse Events (TRAEs) } & \multirow{4}{*}{$\begin{array}{l}\text { Efficac! } \\
\begin{array}{l}\text { ORR } \\
(\%)\end{array}\end{array}$} \\
\hline & & & & & \multirow{2}{*}{\multicolumn{2}{|c|}{$\begin{array}{l}\text { Neutropenia } \\
\text { (\%) }\end{array}$}} & \multirow{2}{*}{\multicolumn{2}{|c|}{$\begin{array}{l}\text { Leukopenia } \\
\text { (\%) }\end{array}$}} & \multirow{2}{*}{\multicolumn{2}{|c|}{$\begin{array}{l}\text { Neuropathy } \\
\text { (\%) }\end{array}$}} & \multirow{2}{*}{\multicolumn{2}{|c|}{$\begin{array}{l}\text { Fatigue } \\
(\%)\end{array}$}} & \\
\hline & & & & & & & & & & & & & \\
\hline & & & & & $\begin{array}{l}\text { All } \\
\text { grades }\end{array}$ & $\begin{array}{l}3 / 4 \\
\text { grade }\end{array}$ & $\begin{array}{l}\text { All } \\
\text { grades }\end{array}$ & $\begin{array}{l}3 / 4 \\
\text { grade }\end{array}$ & $\begin{array}{l}\text { All } \\
\text { grades }\end{array}$ & $\begin{array}{l}3 / 4 \\
\text { grade }\end{array}$ & $\begin{array}{l}\text { All } \\
\text { grades }\end{array}$ & $\begin{array}{l}3 / 4 \\
\text { grade }\end{array}$ & \\
\hline \multirow{4}{*}{$\begin{array}{l}\text { Ibrahim NK } \\
2005\end{array}$} & \multirow{4}{*}{$\begin{array}{l}\text { Phase II } \\
\text { Multicenter }\end{array}$} & 63 & Mixed & $300 \mathrm{mg} / \mathrm{m}^{2}$ & 9 & 51 & 91 & 24 & 64 & 11 & 40 & 13 & 48 \\
\hline & & & & & & & & & & & & & \\
\hline & & 39 & First & $\begin{array}{l}300 \mathrm{mg} / \mathrm{m}^{2} \\
\mathrm{q} 3 \mathrm{w}\end{array}$ & & & & & & & & & 64 \\
\hline & & 24 & Further & $\begin{array}{l}300 \mathrm{mg} / \mathrm{m}^{2} \\
\mathrm{q} 3 \mathrm{w}\end{array}$ & & & & & & & & & 21 \\
\hline \multirow{4}{*}{$\begin{array}{l}\text { Gradishar } \\
\text { WJ } \\
2005\end{array}$} & \multirow{4}{*}{$\begin{array}{l}\text { Phase III } \\
\text { RCT } \\
\text { Multicenter }\end{array}$} & 229 & Mixed & $260 \mathrm{mg} / \mathrm{m}^{2}$ & & 30 & & 7 & & 10 & & 7 & 33 \\
\hline & & & & & & & & & & & & & \\
\hline & & 97 & First & $\begin{array}{l}260 \mathrm{mg} / \mathrm{m}^{2} \\
\mathrm{q} 3 \mathrm{w}\end{array}$ & & & & & & & & & 42 \\
\hline & & 132 & Further & $\begin{array}{l}260 \mathrm{mg} / \mathrm{m}^{2} \\
\mathrm{q} 3 \mathrm{w}\end{array}$ & & & & & & & & & 27 \\
\hline \multirow{2}{*}{$\begin{array}{l}\text { Blum JL } \\
2007\end{array}$} & \multirow{2}{*}{$\begin{array}{l}\text { Phase II } \\
\text { Multicenter }\end{array}$} & 106 & Further & $\begin{array}{l}100 \mathrm{mg} / \mathrm{m}^{2} \\
\mathrm{gw} 3 / 4\end{array}$ & 49 & 18 & 62 & 19 & 25 & 8 & 37 & 5 & 14 \\
\hline & & 75 & Further & $\begin{array}{l}125 \mathrm{mg} / \mathrm{m}^{2} \\
\mathrm{qw} 3 / 4\end{array}$ & 64 & 34 & 66 & 36 & 51 & 19 & 45 & 12 & 16 \\
\hline \multirow{3}{*}{$\begin{array}{l}\text { Guan ZZ } \\
2009\end{array}$} & \multirow{3}{*}{$\begin{array}{l}\text { Phase II } \\
\text { RCT } \\
\text { Multicenter }\end{array}$} & 104 & Further & $260 \mathrm{mg} / \mathrm{m}^{2}$ & 69 & 42 & 64 & 24 & 76 & 7 & 17 & & 54 \\
\hline & & & & & & & & & & & & & \\
\hline & & & & & & & & & & & & & \\
\hline \multirow{2}{*}{$\begin{array}{l}\text { Mirtsching } \\
\text { B } \\
2011\end{array}$} & \multirow{2}{*}{$\begin{array}{l}\text { Phase II } \\
\text { Multicenter }\end{array}$} & 72 & First & $125 \mathrm{mg} / \mathrm{m}^{2}$ & 14 & 11 & 11 & 1.4 & 54 & 8.3 & 58 & 6.9 & 42.2 \\
\hline & & & & पW $3 / 4$ & & & & & & & & & \\
\hline \multirow{4}{*}{$\begin{array}{l}\text { Gradishar } \\
\text { WJ } \\
2009 / 2012\end{array}$} & \multirow{4}{*}{$\begin{array}{l}\text { Phase II } \\
\text { RCT } \\
\text { Multicenter }\end{array}$} & 76 & First & $100 \mathrm{mg} / \mathrm{m}^{2}$ & 80 & 25 & & & 58 & 9 & 34 & 0 & 63 \\
\hline & & & & qw $3 / 4$ & & & & & & & & & \\
\hline & & 74 & First & $\begin{array}{l}150 \mathrm{mg} / \mathrm{m}^{2} \\
\mathrm{qw} 3 / 4\end{array}$ & 92 & 44 & & & 68 & 22 & 47 & 4 & 74 \\
\hline & & 76 & First & $\begin{array}{l}300 \mathrm{mg} / \mathrm{m}^{2} \\
\mathrm{q} 3 \mathrm{w}\end{array}$ & 93 & 44 & & & 73 & 21 & 36 & 5 & 46 \\
\hline \multirow{3}{*}{$\begin{array}{l}\text { Brezden B } \\
2013\end{array}$} & Phase II & 47 & First & $100 \mathrm{mg} / \mathrm{m}^{2}$ & 14.9 & 9 & & & 46.8 & 0 & 70 & & 30 \\
\hline & Multicenter & & & $q w 3 / 4$ & & & & & & & & & \\
\hline & & 76 & First & $\begin{array}{l}100 \mathrm{mg} / \mathrm{m}^{2} \\
\mathrm{qw} 3 / 4\end{array}$ & 17.1 & 9 & & & 57.9 & 0 & 72 & & 28 \\
\hline Ranade AA & Phase II & 55 & Mixed & $220 \mathrm{mg} / \mathrm{m}^{2}$ & & 28 & & & & 1.5 & & & 40 \\
\hline 2013 & RCT & & & & & & & & & & & & \\
\hline & Multicenter & 53 & Mixed & $\begin{array}{l}300 \mathrm{mg} / \mathrm{m}^{2} \\
\mathrm{q} 3 \mathrm{w}\end{array}$ & & 38 & & & & 17 & & & 40 \\
\hline Palumbo R & Phase II & 52 & Further & $260 \mathrm{mg} / \mathrm{m}^{2}$ & 77 & 21.2 & 88.5 & 25 & 48.1 & 5.8 & 27 & 0 & 48.1 \\
\hline 2015 & Multicenter & & & & & & & & & & & & \\
\hline Fabi A & Phase II & 42 & Further & Mixed & 95 & 70 & 95 & 25 & 80 & 12 & 72 & 13 & 23.8 \\
\hline 2015 & $\begin{array}{l}\text { Single- } \\
\text { center }\end{array}$ & 32 & Further & $\begin{array}{l}125 \mathrm{mg} / \mathrm{m}^{2} \\
\mathrm{qw} 3 / 4\end{array}$ & & & & & & & & & 28.1 \\
\hline & & 10 & Further & $\begin{array}{l}260 \mathrm{mg} / \mathrm{m}^{2} \\
q 3 w\end{array}$ & & & & & & & & & 10 \\
\hline Hurria A & Phase II & 39 & Mixed & $100 \mathrm{mg} / \mathrm{m}^{2}$ & 26 & 3 & 36 & 5 & 8 & 0 & 31 & 5 & 31 \\
\hline 2015 & Multicenter & & & & & & & & & & & & \\
\hline
\end{tabular}




\begin{tabular}{|c|c|c|c|c|c|c|c|c|c|c|c|c|c|}
\hline $\begin{array}{l}\text { Andres FT } \\
2015\end{array}$ & $\begin{array}{l}\text { Phase II } \\
\text { RCT } \\
\text { Multicenter }\end{array}$ & 21 & Mixed & $\begin{array}{l}100 \mathrm{mg} / \mathrm{m}^{2} \\
\mathrm{qw} 3 / 4\end{array}$ & 38 & 14 & & & 48 & 5 & 54 & 10 & 38 \\
\hline $\begin{array}{l}\text { Jain MM } \\
2016\end{array}$ & $\begin{array}{l}\text { Phase III } \\
\text { RCT } \\
\text { Multicenter }\end{array}$ & 58 & Mixed & $\begin{array}{l}260 \mathrm{mg} / \mathrm{m}^{2} \\
q 3 \mathrm{w}\end{array}$ & 33 & 21 & 28 & 16 & 60 & 17 & 36 & 7 & 43 \\
\hline $\begin{array}{l}\text { Yamamoto } \\
\text { S } \\
2017\end{array}$ & $\begin{array}{l}\text { Phase II } \\
\text { Multicenter }\end{array}$ & 35 & Mixed & $\begin{array}{l}180 \mathrm{mg} / \mathrm{m}^{2} \\
\mathrm{q} 3 \mathrm{w}\end{array}$ & & 46 & & & & 0 & & & 23 \\
\hline $\begin{array}{l}\text { Tamura K } \\
2017\end{array}$ & $\begin{array}{l}\text { Phase II } \\
\text { RCT } \\
\text { Multicenter }\end{array}$ & 98 & First & $\begin{array}{l}150 \mathrm{mg} / \mathrm{m}^{2} \\
\mathrm{qw} 3 / 4\end{array}$ & 97 & 78 & 96 & 58 & 88 & 22 & 33 & 1 & 61.2 \\
\hline $\begin{array}{l}\text { Bernardo A } \\
2017\end{array}$ & Cohort & $\begin{array}{l}209 \\
68\end{array}$ & $\begin{array}{l}\text { Further } \\
\text { Further }\end{array}$ & $\begin{array}{l}\text { Mixed } \\
125 \mathrm{mg} / \mathrm{m}^{2} \\
\text { qw } 3 / 4\end{array}$ & $\begin{array}{l}9.4 \\
8.7\end{array}$ & $\begin{array}{l}3 \\
1.1\end{array}$ & & & $\begin{array}{l}29.9 \\
17.4\end{array}$ & $\begin{array}{l}2.1 \\
2.2\end{array}$ & $\begin{array}{l}27.4 \\
12\end{array}$ & $\begin{array}{l}1.7 \\
1.1\end{array}$ & $\begin{array}{l}32.1 \\
31.5\end{array}$ \\
\hline & & 121 & Further & $\begin{array}{l}260 \mathrm{mg} / \mathrm{m}^{2} \\
\mathrm{q} 3 \mathrm{w}\end{array}$ & 9.9 & 4.2 & & & 38 & 2.1 & 37.3 & 2.1 & 32.4 \\
\hline $\begin{array}{l}\text { Gennari A } \\
2018\end{array}$ & $\begin{array}{l}\text { Phase II } \\
\text { RCT }\end{array}$ & 83 & First & $\begin{array}{l}150 \mathrm{mg} / \mathrm{m}^{2} \\
\mathrm{q} 2 \mathrm{w}\end{array}$ & 55.4 & 21.7 & & & 69.5 & 8 & & 48.2 & 47 \\
\hline & Multicenter & 86 & First & $\begin{array}{l}100 \mathrm{mg} / \mathrm{m}^{2} \\
\mathrm{qw} 3 / 4\end{array}$ & 68.6 & 27.9 & & & 73.2 & 5.8 & & 46.5 & 54.7 \\
\hline & & 86 & First & $75 \mathrm{mg} / \mathrm{m}^{2} \mathrm{qw}$ & 72.2 & 24.4 & & & 70 & 5.8 & & 10.5 & 44.7 \\
\hline $\begin{array}{l}\text { Marschner } \\
N \\
2018\end{array}$ & $\begin{array}{l}\text { Cohort } \\
\text { Multicenter }\end{array}$ & $\begin{array}{l}697 \\
4991\end{array}$ & $\begin{array}{l}\text { Mixed } \\
\text { Mixed }\end{array}$ & $\begin{array}{l}\text { Mixed } \\
\leq 150 \mathrm{mg} / \mathrm{m}^{2} \\
\text { qw }\end{array}$ & & 4 & & 7.5 & 39.6 & 4.3 & 20.8 & 1.3 & $\begin{array}{l}37.2 \\
39.1\end{array}$ \\
\hline & & 194 & Mixed & $\begin{array}{l}220- \\
260 \mathrm{mg} / \mathrm{m}^{2} \\
q 3 \mathrm{w}\end{array}$ & & & & & & & & & 33 \\
\hline $\begin{array}{l}\text { Hurria A } \\
2019\end{array}$ & $\begin{array}{l}\text { Phase II } \\
\text { Multicenter }\end{array}$ & 40 & Mixed & $\begin{array}{l}100 \mathrm{mg} / \mathrm{m}^{2} \\
\mathrm{qw} 3 / 4\end{array}$ & 44 & 11 & 18 & 3 & 10 & 5 & 55 & 5 & 35 \\
\hline $\begin{array}{l}\text { Ciruelos E } \\
2019\end{array}$ & $\begin{array}{l}\text { Phase II } \\
\text { RCT }\end{array}$ & 16 & First & $\begin{array}{l}100 \mathrm{mg} / \mathrm{m}^{2} \\
\mathrm{qw} 3 / 4\end{array}$ & 37.5 & 0 & 50.1 & 6.3 & 81.3 & 0 & 43.9 & 6.3 & 37.5 \\
\hline & Multicenter & 14 & First & $\begin{array}{l}150 \mathrm{mg} / \mathrm{m}^{2} \\
\mathrm{q} 2 \mathrm{w}\end{array}$ & 18.8 & 0 & 25.1 & 6.3 & 62.6 & 0 & 87.6 & 0 & 12.5 \\
\hline & & 16 & First & $\begin{array}{l}150 \mathrm{mg} / \mathrm{m}^{2} \\
\mathrm{qw} 3 / 4\end{array}$ & 64.2 & 50 & 50 & 28.6 & 78.6 & 35.7 & 64.3 & 14.3 & 42.9 \\
\hline $\begin{array}{l}\text { Hara F } \\
2019\end{array}$ & $\begin{array}{l}\text { Phase II } \\
\text { RCT }\end{array}$ & 48 & Mixed & $\begin{array}{l}180 \mathrm{mg} / \mathrm{m}^{2} \\
\mathrm{q} 3 \mathrm{w}\end{array}$ & 50 & 14.6 & 60.4 & 14.6 & 81.3 & 8.3 & 70.8 & 0 & 37.8 \\
\hline & Multicenter & 45 & Mixed & $\begin{array}{l}220 \mathrm{mg} / \mathrm{m}^{2} \\
\mathrm{q} 3 \mathrm{w}\end{array}$ & 73.3 & 37.7 & 77.8 & 26.6 & 84.4 & 8.9 & 77.8 & 0 & 44.1 \\
\hline & & 47 & Mixed & $\begin{array}{l}260 \mathrm{mg} / \mathrm{m}^{2} \\
\mathrm{q} 3 \mathrm{w}\end{array}$ & 57.4 & 25.4 & 66 & 19.1 & 91.5 & 31.9 & 80.9 & 2.1 & 48.7 \\
\hline $\begin{array}{l}\text { Schmid P } \\
2018 / 2019\end{array}$ & $\begin{array}{l}\text { Phase III } \\
\text { RCT } \\
\text { Multicenter }\end{array}$ & 449 & First & $\begin{array}{l}100 \mathrm{mg} / \mathrm{m}^{2} \\
\mathrm{qw} 3 / 4\end{array}$ & 15 & 8.2 & & & 23 & 2.7 & 44 & 3 & 45.9 \\
\hline
\end{tabular}


Totally $3287 \mathrm{MBC}$ patients treated with nab-paclitaxel monotherapy were included in the current study. In our analysis, there were 1685 (51.26\%) MBC patients who received nab-paclitaxel as first-line therapy, 640 patients (19.47\%) as further line therapy, and the rest 962 MBC patients (29.27\%) as mixed (first or further) line therapy. Furthermore, a majority of MBC patients ( $n=1966,60.40 \%)$ had nab-paclitaxel administered weekly, 1190 MBC patients (36.56\%) had nabpaclitaxel administered triweekly, and $99 \mathrm{MBC}$ patients (3.04\%) with biweekly $150 \mathrm{mg} / \mathrm{m} 2$ nab-paclitaxel administration. Among $1190 \mathrm{MBC}$ patients with triweekly nab-paclitaxel schedule (q3w), despite of 194 patients (16.30\%) being administered at an imprecise reported dosage of $220-260 \mathrm{mg} / \mathrm{m} 2,192$ patients (16.13\%) were administered at the dosage of $300 \mathrm{mg} / \mathrm{m} 2,621$ patients $(52.18 \%)$ were at $260 \mathrm{mg} / \mathrm{m} 2,100$ patients $(8.40 \%)$ were at $220 \mathrm{mg} / \mathrm{m} 2$, and 83 patients (6.97\%) were at $180 \mathrm{mg} / \mathrm{m} 2$. Among $1966 \mathrm{MBC}$ patients with weekly nab-paclitaxel schedule, 956 patients (48.62\%) were administered at the dosage of $100 \mathrm{mg} / \mathrm{m} 2$ qw 3/4, 247 patients (12.56\%) were at $125 \mathrm{mg} / \mathrm{m} 2$ qw 3/4, 186 patients (9.5\%) were at $150 \mathrm{mg} / \mathrm{m} 2$ qw $3 / 4$, and 86 patients (4.4\%) were at $75 \mathrm{mg} / \mathrm{m} 2 \mathrm{qw}$. Still, $491 \mathrm{MBC}$ patients (24.97\%) with weekly nab-paclitaxel monotherapy in the NABUCCO study were reported with administration at $\leq 150 \mathrm{mg} / \mathrm{m} 2$ qw schedule.

Nab-paclitaxel monotherapy safety profiles

All 3287 MBC patients were included in the safety analysis. Neutropenia, leukopenia, peripheral sensory neuropathy and fatigue were the four chosen representative TRAEs of nab-paclitaxel monotherapy.

According to our study, 22 included studies with 31 individual groups reported the incidence of treatment related neutropenia after nab-paclitaxel monotherapy in $3287 \mathrm{MBC}$ patients. After data integration, the overall incidence of all grades neutropenia was $52 \%(95 \% \mathrm{Cl}, 38 \%-66 \%)$, and the incidence of grade $3 / 4$ neutropenia was $24 \%(95 \% \mathrm{Cl}, 16 \%-32 \%)$ (Figure 2A2B). 19 individual groups reported the incidence of chemotherapy related leukopenia with the overall incidence of all-grades leukopenia was $58 \%$ (95\% Cl, 43\%-73\%), and the incidence of grade 3/4 leukopenia was 17\% (95\% Cl, 11\%-24\%) (Figure 2C2D). Together $3287 \mathrm{MBC}$ patients in 31 individual groups reported the incidence of all grades and grade $3 / 4$ peripheral sensory neuropathy. The overall incidence of all grades peripheral sensory neuropathy was $58 \%(95 \% \mathrm{Cl}, 48 \%-68 \%)$, and the incidence of grade $3 / 4$ peripheral sensory neuropathy was only $8 \%$ ( $95 \% \mathrm{Cl}$, $5 \%-10 \%$ ) (Figure 2E2F). 27 individual groups reported the incidence of treatment related fatigue. The overall incidence of all grades fatigue was $49 \%$ (95\% Cl, $41 \%-56 \%)$, and the incidence of grade $3 / 4$ fatigue was $5 \%(95 \% \mathrm{Cl}, 2 \%-8 \%$ ) following nab-paclitaxel administration (Figure $2 \mathrm{G} 2 \mathrm{H}$ ).

\section{Risk factors affecting TRAEs}

According to the results of meta-regression analysis, treatment lines, nab-paclitaxel dosage, nab-paclitaxel schedule did not affect the incidence of all grades neutropenia and grade 3/4 neutropenia. However, grade 3/4 neutropenia occured less frequently in Her-2 negative patients compared with all population (Coef value $=0.063, P=0.046$ ). All grades leukopenia seemed to occur more frequently in MBC patients treated with nab-paclitaxel as further-line therapy (Coef value $=0.366, P=0.056$ ). Treatment lines, patient population, the schedule of nab-paclitaxel administration did not contribute to the development of all grades and grade $3 / 4$ peripheral sensory neuropathy. However, the dosage of nab-paclitaxel monotherapy seemed to be a potential independent risk factor affecting the incidence of grade $3 / 4$ peripheral sensory neuropathy (Coef value $=0.201, P=0.078$ ) as $P$ value almost reached 0.05 . Meanwhile, obvious clinical trend can be noted that grade $3 / 4$ peripheral sensory neuropathy were more frequently recorded in patients with higher nab-paclitaxel dosage group compared with patients with lower dosage group (Figure $3 \mathrm{~A}$ ). Nab-paclitaxel related all grades fatigue occurred more frequently in MBC patients with further-line monotherapy (Coef value=-0.239, $P=0.032$ ).

Nab-paclitaxel monotherapy efficacy outcomes

All 3287 MBC patients were included in the efficacy analysis. ORR, CBR, PFS, and OS were the chosen efficacy endpoints index of nab-paclitaxel monotherapy.

33 individual groups reported the ratio of ORR as the major efficacy of nab-paclitaxel monotherapy. After data integration, the cumulative ratio of ORR was $40 \%$ (95\% Cl, 35\%-45\%). 25 individual groups reported the ratio of CBR with the cumulative ratio being $66 \%$ (95\% Cl, 59\%-73\%). Additionally, complete remission (CR) was noted in 23 individual groups and the cumulative ratio of CR was only $3 \%(95 \% \mathrm{Cl}, 2 \%-5 \%)$. Partial remission (PR) and stable disease (SD) were higher than $\mathrm{CR}$ with the cumulative ratio reaching $38 \%(95 \% \mathrm{Cl}, 32 \%-44 \%)$ and $28 \%(95 \% \mathrm{Cl}, 24 \%-31 \%)$, respectively.

23 individual groups with 2399 MBC patients reported the outcome of PFS after nab-paclitaxel monotherapy. The median PFS ranged from 3.7 to 14.6 months and the overall median PFS was 7.64 months $(95 \% \mathrm{Cl}, 6.89-8.40$ months). The outcome of OS was reported in 14 studies with 17 individual groups containing $2472 \mathrm{MBC}$ patients. The median OS ranged from 15.2 to 44.7 months and the overall median OS was 24.51 months (95\% $\mathrm{Cl}, 21.25-27.78$ months).

Risk factors affecting efficacy outcomes

According to the meta-regression analysis, we found that patients treated with nab-paclitaxel monotherapy in further line would suffer from unfavorable lower ORR (Coef value $=-0.18, P=0.006)$ compared with the patients in other lines. In the subsequent subgroup analysis, the value of $0 R R$ was $48.2 \%$ (95\% $\mathrm{Cl}$, $41 \%-45 \%)$ in patients treated with nab-paclitaxel in the first line, while $40.1 \%(95 \% \mathrm{Cl}, 35.1 \%-45 \%)$ in the mixed line (first or further line), and $27.2 \%$ (95\% $\mathrm{Cl}$, $20.1 \%-34.3 \%$ ) in the further line. Similar result and statistical significance were also obtained concerning to CBR (Coef value=-0.176, $P=0.037$ ), with CBR valued $55.3 \%(95 \% \mathrm{Cl}, 43.2 \%-67.4 \%)$ in the further line, $68.7 \%(95 \% \mathrm{Cl}, 62.5 \%-75 \%)$ in the first line, and $68.8 \%(95 \% \mathrm{Cl}, 63.5 \%-74 \%)$ in the mixed line. The schedule of nab-paclitaxel administration (weekly, biweekly or triweekly) did not affect the ORR (Coef value $=-0.212, P=0.116$ ). However, under the same schedule of nab-paclitaxel administration, patients appeared to have more superior ORR along with the increasing dosage of nab-paclitaxel (Coef value $=0.081$, $P=0.044$ ) (Figure 3B).

Patients who received first line nab-paclitaxel monotherapy was demonstrated to have longer median PFS versus mixed line therapy. The median PFS was 8.01 months ( $95 \% \mathrm{Cl}, 6.83-9.18$ months) in the first line group and 6.55 months (95\% Cl, 5.69-7.4 months) in the mixed line group, respectively. Patients who received further line nab-paclitaxel monotherapy was demonstrated to have rather shorter median OS versus first and mixed line therapy. Median OS values 
were 16.18 months ( $95 \% \mathrm{Cl}, 13.41-18.94$ months), 24.41 months ( $95 \% \mathrm{Cl}, 20.4-28.42$ months), and 28.44 months ( $95 \% \mathrm{Cl}, 12.75-44.13$ months) by further, first, and mixed line therapy, respectively. Similarly, just like the ORR, the schedule of nab-paclitaxel administration (weekly, biweekly or triweekly) did not affect the median PFS (Coef value=-2.77, $P=0.162$ ) and median OS (Coef value=6.27, $P=0.623$ ). Median PFS was 6.94 months (95\% Cl, $5.92-7.96$ months) for weekly and 7.71 months ( $95 \% \mathrm{Cl}, 6.90-8.52$ months) for q3w nab-paclitaxel monotherapy. However, under the same schedule of nab-paclitaxel administration, patients appeared to have longer PFS along with the increasing dosage of nab-paclitaxel (Coef value $=2.68, P=0.03$ ). This significance was not found with respect to the median OS (Coef value $=6.27, P=0.62$ ). Risk factors affecting nab-paclitaxel monotherapy related adverse events and efficacy outcomes were presented in Table 3.

Table 3 Risk factors affecting nab-paclitaxel monotherapy related adverse events and efficacy outcomes by meta-regression analysis

\begin{tabular}{|c|c|c|c|c|c|c|}
\hline Effect Index & Risk Factors & Coef. value & Std. Err. & $\mathrm{T}$ value & $P$ value & 95\% Confidence Interval \\
\hline $\begin{array}{l}\text { Incidence of neutropenia } \\
3 / 4 \text { grade }\end{array}$ & Her2 negative & -0.136 & 0.063 & -2.14 & 0.046 & -0.268 to -0.003 \\
\hline $\begin{array}{l}\text { Incidence of leukopenia } \\
\text { all grades }\end{array}$ & Treatment line & 0.366 & 0.148 & 2.48 & 0.056 & -0.014 to 0.746 \\
\hline $\begin{array}{l}\text { Incidence of neuropathy } \\
3 / 4 \text { grade }\end{array}$ & Nab-paclitaxel dosage & 0.201 & 0.107 & 1.87 & 0.078 & -0.025 to 0.427 \\
\hline $\begin{array}{l}\text { Incidence of fatigue } \\
\text { all grades }\end{array}$ & Treatment line & -0.239 & 0.100 & -2.40 & 0.032 & -0.455 to -0.024 \\
\hline Overall response rate & Treatment line & -0.180 & 0.059 & -3.03 & 0.006 & -0.302 to -0.058 \\
\hline (ORR) & Nab-paclitaxel dosage & 0.171 & 0.081 & 2.11 & 0.044 & 0.005 to 0.338 \\
\hline $\begin{array}{l}\text { Clinical benefit rate } \\
\text { (CBR) }\end{array}$ & Treatment line & -0.176 & 0.077 & -2.29 & 0.037 & -0.340 to -0.012 \\
\hline Progression free survival & Treatment line & 1.398 & 0.635 & 2.20 & 0.045 & 0.036 to 2.760 \\
\hline (PFS) & Nab-paclitaxel dosage & 2.683 & 1.114 & 2.41 & 0.030 & 0.295 to 5.071 \\
\hline $\begin{array}{l}\text { Overall survival } \\
\text { (OS) }\end{array}$ & Treatment line & -18.909 & 8.210 & -2.30 & 0.040 & -36.797 to -1.021 \\
\hline
\end{tabular}

\section{Discussion}

Taxanes are regarded as the most widely used and effective single antitumor agents in the treatment of metastatic breast cancer [38]. Although proven beneficial, the solvent-based toxicities and high incidence of TRAEs restricted the long-term maintenance therapy of paclitaxel and docetaxel. Nab-paclitaxel, a relatively younger member of taxanes family, has gained increasing favor in treating MBC due to its special antitumor characteristics and low toxicities in the past 15 years. Although a proportion of studies reported nab-paclitaxel monotherapy with acceptable safety profiles, higher disease control rates and improved survival in the management of $\operatorname{MBC}[9,16,39,40]$, no systematic data was provided. These limitations could pose a specific question what exactly nab-paclitaxel monotherapy could provide for MBC patients concerning to both TRAEs and clinical benefits.

In our study, the safety profiles of nab-paclitaxel mono-treatment were firstly analyzed. It was known that neutropenia and leukopenia were the most common hematologic adverse events (AEs) of nab-paclitaxel, and some authors even reported the incidence of grade 3/4 neutropenia after nab-paclitaxel monotherapy could be higher than $50 \%$ [14]. Actually, according to our analysis, after integrating each single individual group, the overall incidence of all grades neutropenia and leukopenia was $52 \%$ and $58 \%$, the incidence of grade $3 / 4$ neutropenia and leukopenia was $24 \%$ and $17 \%$, respectively. Furthermore, across to the majority of studies, these hematologic adverse events were generally considered uncomplicated and could be rapidly resolved after treatment interruption, dose reduction and granulocyte colony-stimulating factor (G-CSF) supplement. Although neutropenia and leukopenia were known as dose-limiting, we found the incidence of all grades and grade 3/4 neutropenia and leukopenia were not correlated with the nab-paclitaxel dosage and schedule which indicated neutropenia or leukopenia might not be a dose or schedule-dependent adverse event. We still found that Her2 expression status seemed to be correlated with the incidence of grade 3/4 neutropenia, as the incidence of 3/4 grade neutropenia was significantly lower in the Her2-negative population, however, this reason was still unknown yet.

Peripheral sensory neuropathy is a common and a specific adverse event of taxanes-based therapy. In our analysis, following nab-paclitaxel monotherapy, the overall incidence of all grades peripheral sensory neuropathy was $58 \%$, and grade $3 / 4$ neuropathy was $8 \%$. Interestingly, unlike hematologic AEs, we found that the nab-paclitaxel dosage seemed to be a potential risk factor affecting the incidence of grade $3 / 4$ peripheral sensory neuropathy. Although statistical significance had not obtained, relatively obvious trend could be noted that grade $3 / 4$ peripheral sensory neuropathy were more frequently recorded in patients with higher nab-paclitaxel dosage group compared with patients with lower dosage group. Furthermore, in the fixed weekly or triweekly nab-paclitaxel 
monotherapy, the incidence of grade 3/4 peripheral sensory neuropathy increased if nab-paclitaxel dosage increased. This was in accordance with the results of Ciruelos $\mathrm{E}$ as they found the grade 3 peripheral neuropathy was deemed to be taxanes-related, which is known to be cumulative [35].

Concerning to the efficacy outcomes, our analysis showed nab-paclitaxel monotherapy could provide a $40 \%$ ORR, a $60 \%$ CBR, a median PFS of 7.64 months and a median OS of 24.51 months for the overall population of patients with MBC who received various doses, schedules, and regimens of nab-paclitaxel across all lines of therapy. Still, in MBC patients who received nab-paclitaxel monotherapy in the first line treatment, the nab-paclitaxel efficacy outcomes could be more encouraging with a $48.2 \%$ ORR, a $68.7 \%$ CBR, a median PFS of 8.01 months and a median 0 S of 24.41 months. These results are more superior than the previous real-life study with sizeable sample, which showed a median time to next therapy or death (TNTD) of 6.1 months, a median OS of 17.4 months in patients receiving nab-paclitaxel monotherapy for $\geq$ first-line treatment of MBC [19]. In the current study, we proved treatment line and nabpaclitaxel dosage were the risk factors affecting ORR and median PFS. Patients treated with nab-paclitaxel monotherapy in further or mixed line would endure lower ORR and shorter median PFS compared with patients in first line, and patients appeared to have better ORR or longer PFS along with the increasing dosage of nab-paclitaxel.

Nab-paclitaxel can be used as a triweekly schedule, but it is also justifiable to administer it in various weekly schedules in metastatic MBC. It is particular true that different opinions exist among the experts regarding the optimal schedule of nab-paclitaxel [41]. Findings from a randomized study by Gradishar in 2009 suggested that nab-paclitaxel provided weekly for 3 weeks at a dose of $150 \mathrm{mg} / \mathrm{m} 2$ followed by 1 week of break is more effective in terms of PFS than is $100 \mathrm{mg} / \mathrm{m} 2$ nab-P provided weekly [11]. In our study, we find that weekly administration schedule was more frequently used compared with triweekly schedule. However, according to our analysis, no matter which nab-paclitaxel schedule (weekly, biweekly or triweekly) was chosen, the nab-paclitaxel efficacy outcomes were not affected. The recently published NABUCCO study also showed no differences in terms of clinical activity of nab-paclitaxel according to the schedule used [10]. This finding is of particular interest for the clinical practice. As the schedule of nab-paclitaxel administration is not proven to be corelated with the efficacy outcomes, we suggested that nab-paclitaxel can be safely used with the weekly as well as the triweekly schedule, leaving the choice to the physician according to the patient's needs and preference, with a careful balance between activity and potential toxicity. In a word, nab-paclitaxel offers flexible scheduling. Moreover, treatment line rather than the nab-paclitaxel dosage was demonstrated to be the only risk factor affecting median OS. Indeed, assessment of OS was considered to be more objective than PFS. These findings might partially guide us how nab-paclitaxel could be used in clinical practice on the basis of the current data, that is for patients with higher tumor burden (visceral metastatic disease or $>2$ metastatic lesions) who needs immediate disease control could receive maximum-tolerated dosage of nab-paclitaxel, such as $300 \mathrm{mg} / \mathrm{m} 2 \mathrm{q} 3 \mathrm{w}$ or $150 \mathrm{mg} / \mathrm{m} 2 \mathrm{qw} 3 / 4$. These regimens would bring patients with better ORR and longer PFS, but the overall survival might not be changed.

In this systematic review and meta-analysis, we demonstrated nab-paclitaxel mono-chemotherapy was a low-toxic and effective strategy in palliative management for MBC patients. Both weekly and triweekly nab-paclitaxel mono-chemotherapy were proved to be effective for MBC. Higher ORR, longer PFS and OS would be achieved in patients treated with nab-paclitaxel as first line. Increasing nab-paclitaxel dosage would result in better tumor control (higher ORR and PFS), however, changing nab-paclitaxel schedule had no benefit on ameliorating the survival outcomes.

Finally, limitations still existed in this research : (1)Part of studies we included without high quality relatively. (2) Lack of data from multi-center randomized clinical trials. (3) More databases or other resources need to be searched to improve the accuracy of results. In future, We will carry out further research to prove whether our conclusion is correct.

\section{Conclusions}

According to our research, the following conclusions can be drawn: (1) Both weekly and triweekly nab-paclitaxel mono-chemotherapy were proved to be effective for MBC with generally reasonable toxicity profiles. (2) Higher ORR, longer PFS and OS would be achieved in patients treated with nab-paclitaxel as first line. (3) Increasing nab-paclitaxel dosage would result in better tumor control (higher ORR and PFS).(4) Changing nab-paclitaxel schedule had no benefit on ameliorating the survival outcomes. In general, nab-paclitaxel has a distinct advantage in the treatment of MBC, which can be a superior option in clinical strategy.

\section{Abbreviations}

MBC

metastatic breast cancer

ORR

overall response rate

CBR

clinical benefit rate

PFS

progression free survival

OS

overall survival

TTP

time to progression

TRAES

treatment-related adverse events

FDA 
Food and Drug Administration

QOL

quality of life

PRISMA

Preferred Reporting Items for Systematic Reviews and Meta-Analyses

ASCO

American Society of Clinical Oncology

ESMO

European Society of Medical Oncology

SABCS

San Antonio Breast Cancer Symposium

RCTs

randomized controlled trials

MINORS

Methodological Index for Non-randomized Studies

$\mathrm{Cl}$

confidence interval

$\mathrm{CR}$

complete remission

PR

partial remission

SD

stable disease

AEs

adverse events

G- CSF

$\mathrm{H}$ - granulocyte colony-stimulating factor

TNTD

time to next therapy or death

\section{Declarations}

Ethics approval and Consert to participate Not applicable.

Consert for publication Not applicable.

Availability of data and materials All data generated or analysed during this study are included in this published article.

Competing interest None of the authors have the potential competing interests in this manuscript.

Funding The analysis and interpretation of data are funded by the Pyramid Talented Personnel Project of Changzheng Hospital which CS applied .

\section{Authors' contributions}

Research design CS.

Systematic search and Study selection CS and HL.

Data analysis and interpretation CS.

Initial manuscript writing CS, HL, SZ and WZ.

Manuscript revision All authors.

Final approval of manuscript All authors.

Acknowledgements Not applicable.

\section{References}

1. O'Shaughnessy J, Gradishar WJ, Bhar P, et al. Nab-paclitaxel for first-line treatment of patients with metastatic breast cancer and poor prognostic factors: a retrospective analysis. Breast Cancer Res Treat. 2013, 138(3):829-837.

2. Cronin KA, Lake AJ, Scott S, et al. Annual Report to the Nation on the Status of Cancer, Part I: National Cancer Statistics. Cancer. 2020,126(10):2225-2249. 
3. Ghersi D, Willson ML, Chan MM, et al. Taxane-containing regimens for metastatic breast cancer. Cochrane Database Syst Rev. 2015, $2015(6)$ :CD003366. doi: $10.1002 / 14651858$.

4. Vishnu P, Roy V. Safety and Efficacy of nab-Paclitaxel in the Treatment of Patients with Breast Cancer. Breast Cancer (Auckl). 2011, 5:53-65.

5. Cortazar P, Justice R, Johnson J, et al. US Food and Drug Administration approval overview in metastatic breast cancer. J Clin Oncol. 2012, 30(14):17051711.

6. ten Tije AJ, Verweij J, Loos WJ, et al. Pharmacological effects of formulation vehicles: implications for cancer chemotherapy. Clin Pharmacokinet. 2003, 42(7):665-685.

7. Martín M. nab-Paclitaxel dose and schedule in breast cancer. Breast Cancer Res. 2015, 17(1):81-90.

8. Desai N, Trieu V, Yao R, et al. Evidence of greater antitumor activity of Cremophor-free nanoparticle albumin-bound (nab) paclitaxel (Abraxane) compared to Taxol: Role of a novel albumin transporter mechanism. Presented at the 26th Annual San Antonio Breast Cancer Symposium, San Antonio, TX, December 3-6, 2003.

9. Ibrahim NK, Samuels B, Page R, et al. Multicenter phase II trial of ABI-007, an albumin-bound paclitaxel, in women with metastatic breast cancer. J Clin Oncol. 2005, 23(25):6019-6026.

10. Marschner N, Salat C, Söling U, et al. Final Effectiveness and Safety Results of NABUCCO: Real-World Data From a Noninterventional, Prospective, Multicenter Study in 697 Patients With Metastatic Breast Cancer Treated With nab-Paclitaxel. Clin Breast Cancer. 2018, 18(6):e1323-e1337.

11. Gradishar WJ, Krasnojon D, Cheporov S, et al. Significantly longer progression-free survival with nab-paclitaxel compared with docetaxel as first-line therapy for metastatic breast cancer. J Clin Oncol. 2009, 27(22):3611-3619.

12. Gradishar WJ, Tjulandin S, Davidson N, et al. Phase III trial of nanoparticle albumin-bound paclitaxel compared with polyethylated castor oil-based paclitaxel in women with breast cancer. J Clin Oncol. 2005, 23(31):7794-7803.

13. Tamura K, Inoue K, Masuda N, et al. Randomized phase II study of nab-paclitaxel as first-line chemotherapy in patients with HER2-negative metastatic breast cancer. Cancer Sci. 2017, 108(5):987-994.

14. Rugo HS, Barry WT, Moreno-Aspitia A, et al. Randomized Phase III Trial of Paclitaxel Once Per Week Compared With Nanoparticle Albumin-Bound NabPaclitaxel Once Per Week or Ixabepilone With Bevacizumab As First-Line Chemotherapy for Locally Recurrent or Metastatic Breast Cancer: CALGB 40502/NCCTG N063H (Alliance). J Clin Oncol. 2015, 33(21):2361-2369.

15. S Dent, J Fraser, N Graham, et al. Clinical outcomes of women with metastatic breast cancer treated with nab-paclitaxel: experience from a single academic cancer centre. Curr Oncol. 2013, 20(1):24-29.

16. Gradishar WJ, Krasnojon D, Cheporov S, et al. Phase II trial of nab-paclitaxel compared with docetaxel as first-line chemotherapy in patients with metastatic breast cancer: final analysis of overall survival. Clin Breast Cancer. 2012, 12(5):313-321.

17. De Luca R, Profita G, Cicero G. Nab-paclitaxel in pretreated metastatic breast cancer: evaluation of activity, safety, and quality of life. Onco Targets Ther. 2019,12:1621-1627.

18. Mahtani RL, Parisi M, Glück S, et al. Comparative effectiveness of early-line nab-paclitaxel vs. paclitaxel in patients with metastatic breast cancer: a US community-based real-world analysis. Cancer Manag Res. 2018, 10:249-256.

19. Liang C, Li L, Fraser CD, et al. The treatment patterns, efficacy, and safety of nab (®)-paclitaxel for the treatment of metastatic breast cancer in the United States: results from health insurance claims analysis. BMC Cancer. 2015, 15:1019-1030.

20. Moher D, Liberati A, Tetzlaff J, et al. Preferred reporting items for systematic reviews and meta-analyses: the PRISMA statement. BMJ. 2009;339:b2535.

21. Blum JL, Savin MA, Edelman G, et al. Phase II study of weekly albumin-bound paclitaxel for patients with metastatic breast cancer heavily pretreated with taxanes. Clin Breast Cancer. 2007, 7(11):850-856.

22. Guan ZZ, Li QL, Feng FY. Superior efficacy of a Cremophor-free albumin-bound paclitaxel compared with solvent-based paclitaxel in Chinese patients with metastatic breast cancer. Asia Pac J Clin Oncol. 2009, 5:165-174.

23. Mirtsching B, Cosgriff T, Harker G, et al. A phase II study of weekly nanoparticle albumin-bound paclitaxel with or without trastuzumab in metastatic breast cancer. Clin Breast Cancer. 2011, 11(2):121-128.

24. Brezden CB, Cantin G, Younus J, et al. An open-label, phase Il study of weekly nab-paclitaxel as first-line therapy for patients (pts) with metastatic breast cancer (MBC): Safety update. J Clin Oncol. 2016. DOI: 10.1200/jco.2010.28.15_suppl.1127.

25. Ranade AA, Bapsy PP, Nag S, et al. A multicenter phase II randomized study of Cremophor-free polymeric nanoparticle formulation of paclitaxel in women with locally advanced and/or metastatic breast cancer after failure of anthracycline. Asia Pac J Clin Oncol. 2013, 9(2):176-181.

26. Palumbo R, Sottotetti F, Trifirò G, et al. Nanoparticle albumin-bound paclitaxel (nab-paclitaxel) as second-line chemotherapy in HER2-negative, taxanepretreated metastatic breast cancer patients: prospective evaluation of activity, safety, and quality of life. Drug Des Devel Ther. 2015,9:2189-2199.

27. Fabi A, Giannarelli D, Malaguti P, et al. Prospective study on nanoparticle albumin-bound paclitaxel in advanced breast cancer: clinical results and biological observations in taxane-pretreated patients. Drug Des Devel Ther. 2015, 9: 6177-6183.

28. Hurria A, Blanchard MS, Synold TW, et al. Age-Related Changes in Nanoparticle Albumin-Bound Paclitaxel Pharmacokinetics and Pharmacodynamics: Influence of Chronological Versus Functional Age. Oncologist. 2015, 20(1):37-44.

29. Andres FT, Katherine EV, Vandana GA, et al. TBCRC 019: An open label, randomized, phase II trial of nanoparticle albumin-bound paclitaxel (nab-PAC or Abraxane ${ }^{\circledR}$ ) with or without the anti-death receptor 5 (DR5) monoclonal antibody tigatuzumab in patients with metastatic triple negative breast cancer. Clin Cancer Res. 2015, 21(12): 2722-2729.

30. Jain MM, Gupte SU, Patil SG, et al. Paclitaxel injection concentrate for nanodispersion versus nab-paclitaxel in women with metastatic breast cancer: a multicenter, randomized, comparative phase II/III study. Breast Cancer Res Treat. 2016, 156:125-134.

Page $12 / 15$ 
31. Yamamoto S, Maeda N, Nagashima Y, et al. A phase II, multicenter, single-arm study of tri-weekly low-dose nanoparticle albumin-bound paclitaxel chemotherapy for patients with metastatic or recurrent breast cancer. Breast Cancer. 2017, 24(6):783-789.

32. Bernardo A, Palumbo R, Pedersini R, et al. Nab-Paclitaxel in Advanced HER2-negative Breast Cancer Patients: Efficacy and Safety Beyond Clinical Trials. Clin Breast Cancer. 2017, 17(6):433-440.

33. Gennari A, Sun Z, Hasler-Strub U, et al. A randomized phase II study evaluating different maintenance schedules of nab-paclitaxel in the first-line treatment of metastatic breast cancer: final results of the IBCSG 42-12/BIG 2-12 SNAP trial. Ann Oncol. 2018, 29(3):661-668.

34. Hurria A, Soto-Perez-de-Celis E, Blanchard S, et al. A Phase II Trial of Older Adults With Metastatic Breast Cancer Receiving nab-Paclitaxel: Melding the Fields of Geriatrics and Oncology. Clin Breast Cancer. 2019, 19(2):89-96.

35. Ciruelos E, Apellániz-Ruiz M, Cantos B, et al. A Pilot, Phase II, Randomized, Open-Label Clinical Trial Comparing the Neurotoxicity of Three Dose Regimens of Nab-Paclitaxel to That of Solvent-Based Paclitaxel as the First-Line Treatment for Patients with Human Epidermal Growth Factor Receptor Type 2Negative Metastatic Breast Cancer. Oncologist. 2019, 24(11):e1024-e1033.

36. Hara F, Kitada M, Takahashi M, et al. Randomized, optimal dose-finding, phase Il study of tri-weekly nab-paclitaxel in patients with metastatic breast cancer (ABROAD). J Clin Oncol. 2019, DOI: 10.1200/JC0.2019.37.15_suppl.1070.

37. Schmid P, Rugo HS, Adams S, et al. Atezolizumab plus nab-paclitaxel as first-line treatment for unresectable, locally advanced or metastatic triple-negative breast cancer (IMpassion130): updated efficacy results from a randomised, double-blind, placebo-controlled, phase 3 trial. Lancet Oncol. 2020, 21(1):4459.

38. Beslija S, Bonneterre J, Burstein H, et al. Second consensus on medical treatment of metastatic breast cancer. Ann Oncol. 2007, 18(2):215-225.

39. Paoletti C, Li YF, Muñiz MC, et al. Significance of Circulating Tumor Cells in Metastatic Triple-Negative Breast Cancer Patients within a Randomized, Phase II Trial: TBCRC 019. Clin Cancer Res. 2015, 21(12):2771-2779.

40. Aapro M, Tjulandin S, Bhar P, et al. Weekly nab-paclitaxel is safe and effective in $\geq 65$ years old patients with metastatic breast cancer: a post-hoc analysis. Breast. 2011, 20(5):468-474.

41. Cazzaniga ME, Ciruelos E, Fabi A, et al. Metastatic or locally advanced breast cancer patients: towards an expert consensus on nab-paclitaxel treatment in HER2-negative tumours-the MACBETH project. Cancer Chemother Pharmacol. 2019, 83(2):301-318.

\section{Figures}

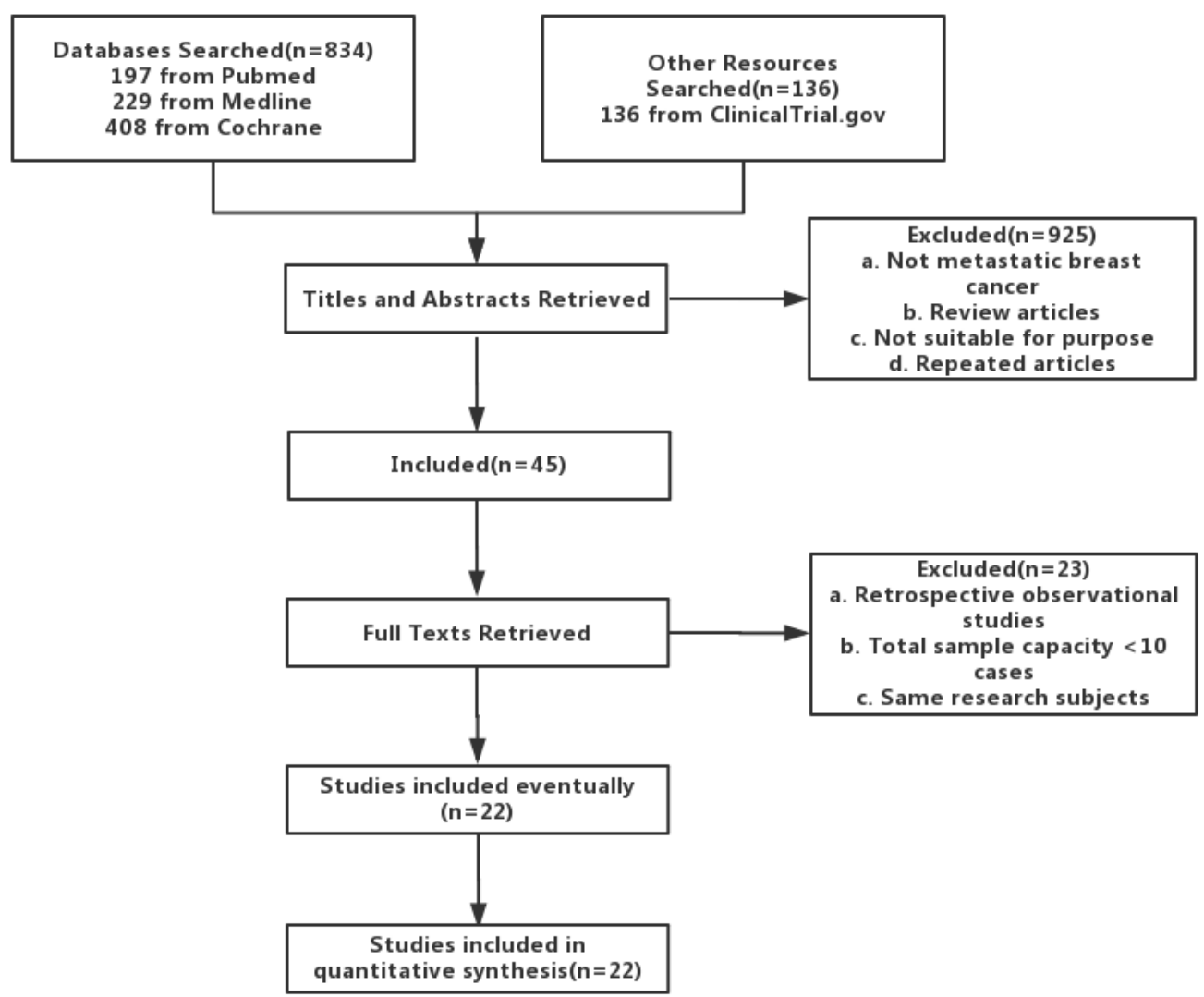

Figure 1 
Study retrieval flow diagram

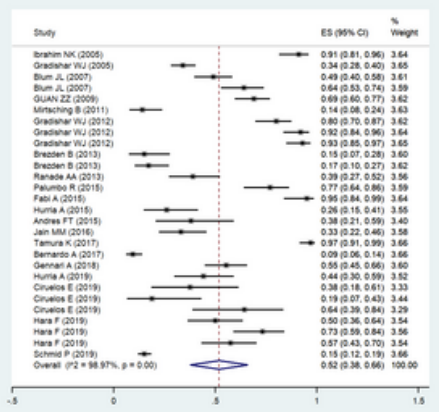

A

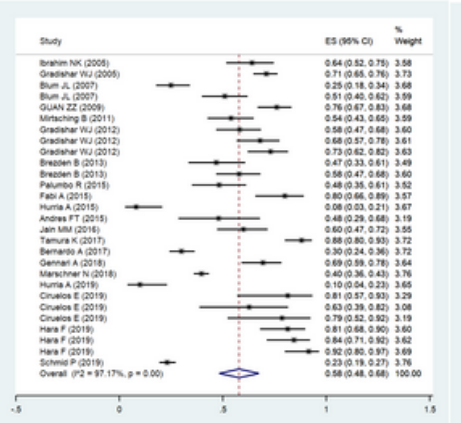

E

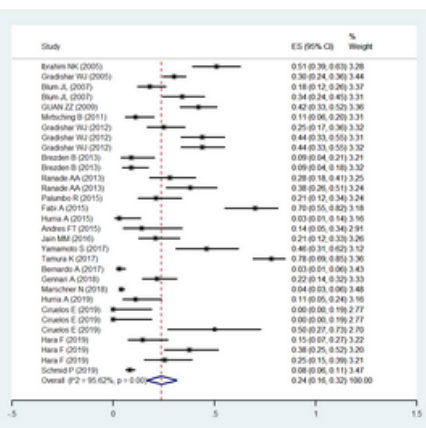

B

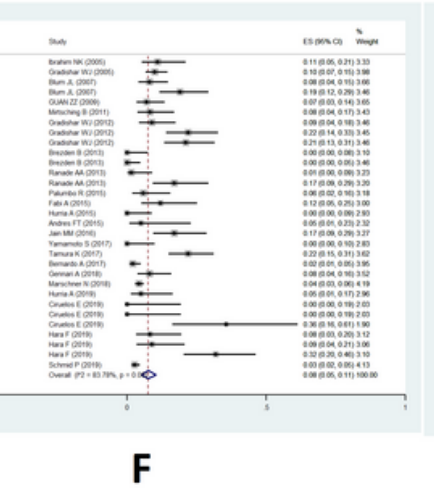

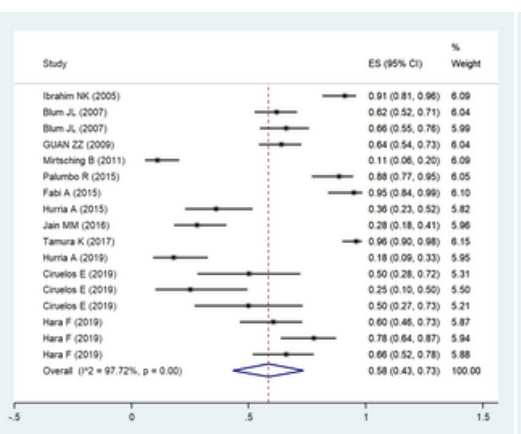

C

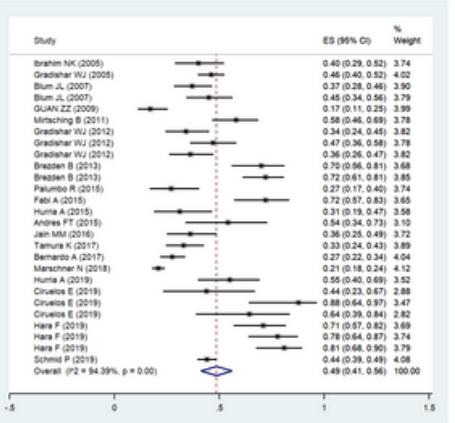

G

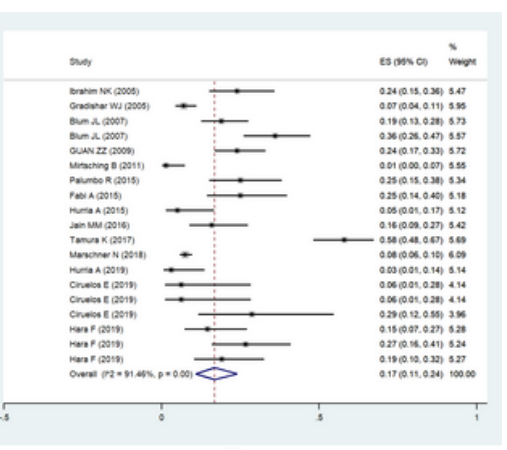

D

\section{Figure 2}

A Incidence of all grades neutropenia B Incidence of grade 3/4 neutropenia C Incidence of all grades leukopenia D Incidence of grade 3/4 leukopenia $E$ Incidence of all grades peripheral sensory neuropathy $\mathrm{F}$ Incidence of grade $3 / 4$ peripheral sensory neuropathy $\mathrm{G}$ Incidence of all grades fatigue $\mathrm{H}$ Incidence of grade $3 / 4$ fatigue

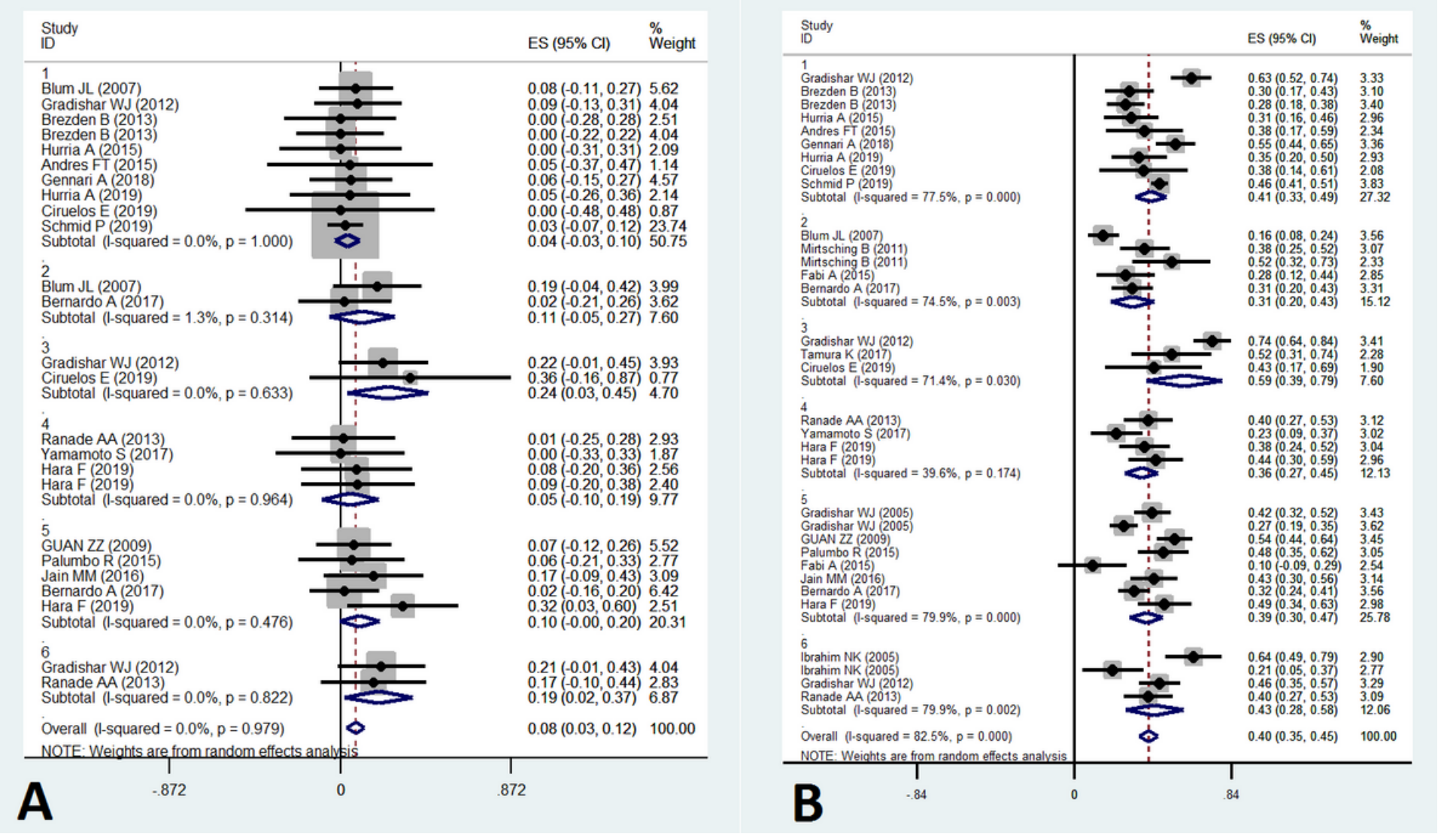

Figure 3 
A Incidence of grade $3 / 4$ peripheral sensory neuropathy related to different nab-paclitaxel dosage B Overall response rate related to different nab-paclitaxel dosage

\section{Supplementary Files}

This is a list of supplementary files associated with this preprint. Click to download.

- PRISMAchecklist.doc 